\title{
Effects of plant availability and habitat size on the coexistence of two competing parasitoids in a tri-trophic food web of canola, diamondback moth and parasitic wasps
}

\author{
Mahmoud Soufbaf $^{\mathrm{a}}$, Yaghoub Fathipour ${ }^{\mathrm{a}, *}$, Cang Hui ${ }^{\mathrm{b}}$, Javad Karimzadeh $^{\mathrm{c}}$ \\ a Department of Entomology, Faculty of Agriculture, Tarbiat Modares University, P.O. Box 14115-336, Tehran, Iran \\ ${ }^{\mathrm{b}}$ Center for Invasion Biology, Department of Botany and Zoology, Stellenbosch University, Matieland 7602, South Africa \\ ${ }^{c}$ Department of Plant Protection, Isfahan Research Centre for Agriculture and Natural Resources, Isfahan, Iran
}

\section{A R T I C L E I N F O}

\section{Article history:}

Received 13 January 2012

Received in revised form 30 May 2012

Accepted 30 June 2012

\section{Keywords:}

Plutella xylostella

Resource availability

Competition

Coexistence

Herbivore starvation

Cellular automata

\begin{abstract}
A B S T R A C T
Using experiments and spatial modeling, we here show the conditions for two competing parasitoids to coexist under two levels of plant availability in a tri-trophic food web of canola, diamondback moth (Plutella xylostella) and two parasitic wasps (Diadegma semiclausum and Cotesia vestalis) that target the same life stage of the herbivore diamondback moth. Plant availability had a significant effect on both the abundances of parasitoids and the herbivore. However, parasitoid abundances were not different with or without the occurrence of other parasitoid species, suggesting no effect of parasitoid coexistence on their abundances. Using a three-species Lotka-Volterra model, we confirmed the competitive exclusion of species with low consumption rate (=initial population size $\times$ per capita consumption rate) in a wellmixed habitat patch. We further built a cellular automaton (CA) to explore the minimum habitat size that ensures the coexistence of these two parasitoids, assuming different searching efficiencies (i.e. different radiuses of searching area and movement abilities for locating the host). With the in-patch dynamics portrayed by the three-species Lotka-Volterra model, the CA model demonstrated the possibility for the two parasitoids to coexist when the number of habitat patches reached a certain number. This research thus highlights the importance of both plant availability and habitat size to the dynamics and stability of the tri-trophic food web.
\end{abstract}

(ㄷ) 2012 Elsevier B.V. All rights reserved.

\section{Introduction}

Plants can indirectly affect the natural enemies (e.g. parasitoids and predators) of herbivores by directly regulating the density of the herbivores (known as the density-mediated indirect interaction; Werner and Peacor, 2003). For instance, the nutrient content in plants can affect herbivore vitality and leads to cascaded effects on the dynamics and coexistence of the herbivore's natural enemies (Bottrell et al., 1998). The density regulation could be specifically strong if the herbivore is a specialist or if the plants are rare and patchily distributed. Indeed, this indirect interaction has been a main driver of ecological variation in many communities (Ohgushi, 2007). Plant density, diversity and patch size have been identified as factors that can directly regulate herbivore density and thus indirectly regulate the dynamics of parasitoids and predators. However, the effect of plant patch size on herbivore density has yielded

\footnotetext{
* Corresponding author. Tel.: +98 21 48292301; fax: +98 2148292200.

E-mail address: fathi@modares.ac.ir (Y. Fathipour).
}

inconsistent results in literature (Bach, 1988). Systematic analysis is also lacking on the indirect interactions between plant availability and the parasitoids of herbivores, as well as the stability and coexistence of this tri-trophic system. It is even more difficult to forecast whether these indirect interactions will enhance or reduce the population size of herbivores and parasitoids, which is especially relevant when the focal species is a direct target of biological control.

Competition between multiple parasitoids is common in such tri-trophic systems (McGlade, 1999; Schoener, 1982; Walls, 1990). Classical competition theory predicts the exclusion of particular parasitoids when they compete for a single resource (Chesson and Case, 1986); that is, competing species cannot coexist unless mechanisms exist to allow the resource being partitioned (Bonsall et al., 2002). Many mechanisms have been identified to promote the coexistence of two parasitoids when feeding on a single host, such as temporal and spatial partitioning of resource (Comins and Hassell, 1996) and differences in attack rate and emergence rate, as well as differences in searching and dispersal abilities (Amarasekare, 2007; Hastings, 1980). These mechanisms of niche 
Plant unlimited treatment

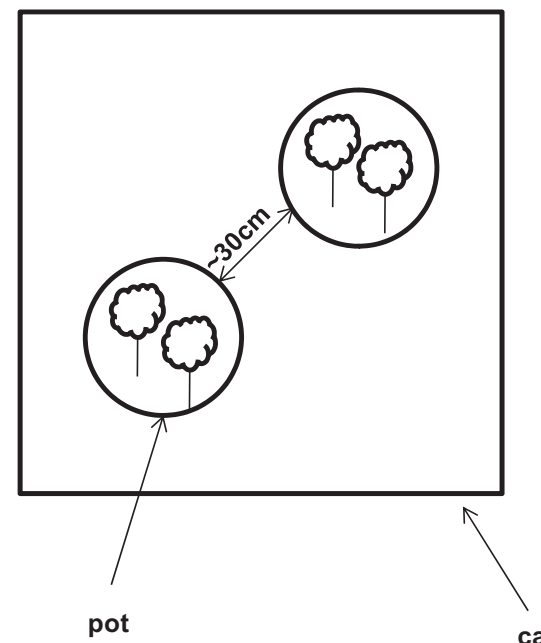

Plant limited treatment

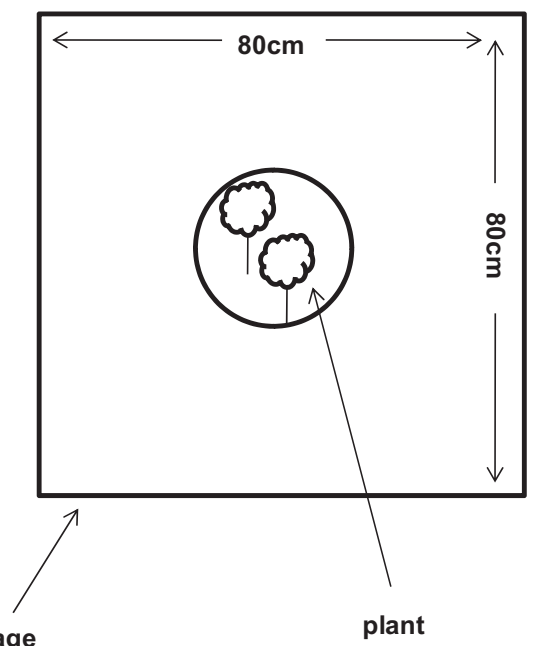

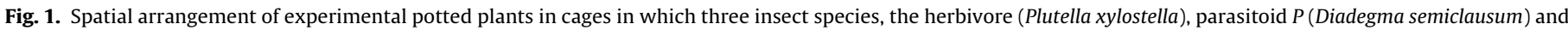
parasitoid $Q$ (Cotesia vestalis) coexist with canola plants for 8-9 herbivore generations (25 weeks) under fixed environmental conditions.

differentiation and partition can be considered as stabilizing forces to ensure the long-term coexistence of multiple competing species (Adler et al., 2007).

Here, using a laboratory experiment and an extended lattice model, we demonstrate how two parasitoids compete and coexist on potted plants in cages, even when the coexistence on a single patch (e.g. a leaf) is predicted unfeasible. We hypothesize that the coexistence of the two parasitoids is made possible because the plants and also leaves are patchily distributed at micro scales. The patchy habitat formed by different levels of plant availability could provide conditions for coexistence. We demonstrate this hypothesis using cellular automata (CA). Our study is a joint research of both greenhouse trails and mathematical modeling that support the observed coexistence due to the spatial partition of hosts. Given the importance of maintaining high biodiversity and enhancing the guild functionality of parasitoids in food webs, our research highlights how changes in plant availability could cause periodic starvations to the herbivores and, therefore, indirectly affect the dynamics, stability, and spatial distributions of the two parasitoids attacking the common host.

\section{Methods}

\subsection{Study species}

Our system comprises a guild of two parasitic wasps, the specialist Diadegma semiclausum (Hellén) (Hymenoptera: Ichneumonidae) and the oligophagous Cotesia vestalis (Haliday) (Hymenoptera: Braconidae), and a specialist herbivore, the diamondback moth (DBM), P. xylostella (L.) (Lepidoptera: Plutellidae) that feeds on the host plants of a canola cultivar (Brassica napus cv. $\mathrm{SLM}_{046}$ ). Both wasps are solitary, koinobiont and endoparasitoids of DBMs; they oviposit into the larvae of all four instars of DBMs (Talekar and Yang, 1993).

\subsection{Experimental design}

A canola cultivar, $\mathrm{SLM}_{046}$, was used in greenhouse trials. It is the most suitable host for DBM amongst 10 tested canola cultivars (Soufbaf et al., 2010). Seeds were collected from the Seed and Plant Improvement Institute (Karaj, Iran) and used for raising plants in field soil without the use of any fertilizers under greenhouse conditions $\left(25 \pm 5^{\circ} \mathrm{C}, 60 \pm 10 \% \mathrm{RH}\right.$, and LD 16:8 h). The DBM larvae and pupae were originally collected from cabbages grown in the horticulture fields at Tehran University. The colonies of $D$. semiclausum and $C$. vestalis were introduced to DBM larvae in ventilated Perspex cages $(30 \mathrm{~cm} \times 30 \mathrm{~cm} \times 30 \mathrm{~cm})$ in the laboratory at $25 \pm 2{ }^{\circ} \mathrm{C}$, and the 4 th generation adult parasitoids were used in further treatments.

A cage experiment on the effect of resource limitation on population dynamics was implemented using the canola cultivar under two different levels of availability: a low resource availability (plant limited treatment), where new plants were provided in each cage every 8 days, and a high resource availability (plant un-limited treatment), where new plants were put in cages before the complete consumption of all leaves by DBM larvae (usually every 3 days in high herbivore densities). For each plant treatment three scenarios of natural enemies were established: DBM $+D$. semiclausum, $\mathrm{DBM}+\mathrm{C}$. vestalis and $\mathrm{DBM}+$ both parasitoids, and each scenario was then replicated four times. Each experiment started with 5 pairs (male and female) of 1-day-old DBM adults; with pots each containing two 4-week-old plants and aqueous sugar solution (10\%) in each cage. One pot was used for plant limited treatment, and two pots for plant unlimited treatment (Fig. 1). After 20 days when DBMs of all stages have established (DBM has overlapping generations), two 2-day-old mated females of each parasitoid with one respective male were released to each parasitoid treatment cage. The cages $(80 \mathrm{~cm} \times 80 \mathrm{~cm} \times 100 \mathrm{~cm})$ were maintained at constant environmental conditions $\left(25 \pm 1^{\circ} \mathrm{C} ; 60 \pm 5 \% \mathrm{RH}\right.$; L:D 16:8 h). The experiment was run for 25 weeks, which equals nearly 8-9 completed generations of DBM. Populations were monitored weekly by counting live adults in each cage. It was evident that, when the density of DBMs is high in the limited plant treatment, individuals can consume all the leaves a few days before replacement; that is, the specialist herbivores suffered a short starvation period mimicking food scarcity after canola harvest season or when multicultural crops are planted.

\subsection{Statistical analysis}

Multivariate general linear models with estimated marginal means (EMMEANS) were used to assess the effects of plant availability and parasitoid coexistence (i.e. the three establishment scenarios of natural enemies) on both herbivore 
Table 1

Description and values of model parameters.

\begin{tabular}{|c|c|c|c|}
\hline Symbol & Description & Value & Source \\
\hline$r$ & Intrinsic growth rate of herbivore $H$ & 0.304 day $^{-1}$ & Soufbaf et al. (2010) \\
\hline$K$ & Carrying capacity of environment & Varied & This experiment \\
\hline$m_{p}$ & Death rate of parasitoid $P$ & $0.03 \mathrm{day}^{-1}$ & Soufbaf et al. (2012) \\
\hline$m_{q}$ & Death rate of parasitoid $Q$ & 0.078 day $^{-1}$ & Nofemela (2004) \\
\hline$\gamma_{p}$ & Attack rate of parasitoid $P$ & $4 \mathrm{~h}^{-1}$ & Wang and Keller (2002) \\
\hline$\gamma_{q}$ & Attack rate of parasitoid $Q$ & $2.5 \mathrm{~h}^{-1}$ & Wang and Keller (2002) \\
\hline$\mu_{p}$ & Handling time of parasitoid $P$ & 0.003 day & Hemerik (2007) \\
\hline$\mu_{q}$ & Handling time of parasitoid $Q$ & 0.032 day & Mitsunaga et al. (2004) \\
\hline$\alpha_{p q}$ & Competition coefficient from parasitoid $Q$ on $P$ & 0.42 & This experiment \\
\hline$\alpha_{q p}$ & Competition coefficient from parasitoid $P$ on $Q$ & 0.58 & This experiment \\
\hline$e_{p}$ & Emigration coefficient of parasitoid $P$ & 0.01 & Estimated from Wang and Keller (2002) \\
\hline$e_{q}$ & Emigration coefficient of parasitoid $Q$ & 0.02 & Estimated from Wang and Keller (2002) \\
\hline$n_{p}$ & Number of neighbors for parasitoid $P$ & 8 & Estimated from Wang and Keller (2002) \\
\hline$n_{q}$ & Number of neighbors for parasitoid $Q$ & 4 & Estimated from Wang and Keller (2002) \\
\hline
\end{tabular}

and parasitoid abundances. Correlations were conducted using Pearson product-moment coefficient $(r)$ with a significance level of $\alpha=0.05$, to identify relationships between two parasitoids densities within different simulated habitats. All statistical analyses were conducted using SPSS 16 (SPSS, 2008).

\subsection{The model}

Since the host-parasitoid system has overlapping generations, we analyzed the population dynamics of this tri-trophic system using the following three-species Lotka-Volterra model based on ordinary differential equations (ODE) that incorporate Holling's (1959) type II functional response (Amarasekare, 2002):

$$
\begin{aligned}
& \frac{d H}{d T}=r H\left(1-\frac{H}{K(R)}\right)-\frac{\gamma_{p} P H}{1+\gamma_{p} \mu_{p} H}-\frac{\gamma_{q} Q H}{1+\gamma_{q} \mu_{q} H} \\
& \frac{d P}{d T}=\frac{\gamma_{p} P H}{1+\gamma_{p} \mu_{p} H}-m_{p} P-\alpha_{p q} Q P \\
& \frac{d Q}{d T}=\frac{\gamma_{q} Q H}{1+\gamma_{q} \mu_{q} H}-m_{q} Q-\alpha_{q p} P Q
\end{aligned}
$$

where $r$ stands for the intrinsic rate of increase and $K(R)$ stands for the carrying capacity of herbivores under different plant resource availabilities $(R)$. Subscripts ( $p$ and $q$ ) indicate parameters associated with specific species $(D$. semiclausum and $C$. vestalis, respectively). The values of all parameters were estimated either from our experiments or taken from literature (Table 1). To check for the sensitivity to initial conditions, we solved the above ODEs numerically with the initial population size of each species assigned from 0 to 1 with a step size of 0.05 .

To examine how the number of patches (leaves) affects the coexistence and spatial distribution of the two parasitoids, we developed a stochastic cellular automaton (CA) under different numbers of patches on a two dimensional habitat with a periodic boundary condition (Hui and Li, 2003, 2004; Ramanantoanina et al., 2011):

$$
\begin{aligned}
& H_{t+1}[i, j]=H_{t}[i, j]+\varepsilon \times \frac{\partial H_{t}[i, j]}{\partial t} \\
& P_{t+1}[i, j]=P_{t}[i, j]+\varepsilon \times \frac{\partial P_{t}[i, j]}{\partial t}-e_{p} P_{t}[i, j]+\frac{e_{p}}{n_{p}} \sum_{(x, y) \in \Omega_{P}} P_{t}[x, y] \\
& Q_{t+1}[i, j]=Q_{t}[i, j]+\varepsilon \times \frac{\partial Q_{t}[i, j]}{\partial t}-e_{q} Q_{t}[i, j]+\frac{e_{q}}{n_{q}} \sum_{(x, y) \in \Omega_{Q}} P_{t}[x, y]
\end{aligned}
$$

where $\varepsilon$ represents the Euler coefficient for calculating the numerical solutions to the full system (Eq. (1)) $(\varepsilon=0.1) ; \Omega_{P}$ indicates the neighboring patches of patch $(x, y)$ for parasitoid $P$ and $\Omega_{Q}$ for $Q$; $e$ and $n$ stand for the emigration rate and the movement range (the number of patches the parasitoid can move during one time step) for specific parasitoid. The ODEs and CAs were solved numerically using Mathematica 8.0 (Wolfram Research Inc.). The in-patch dynamics (the derivative terms above) was governed by the ODEs in Eq. (1), and the movement of DBM between patches was ignored.

\subsection{Parameterization}

This experiment was the last part of three consecutive experiments. The intrinsic population growth rate $(r)$ of the herbivore in the absence of parasitoid was estimated from the first experiment (Soufbaf et al., 2010), and the mortality of parasitoid $P\left(m_{p}\right)$ was estimated from the second experiment (Soufbaf et al., 2012). The carrying capacity $(K)$ and the per capita effects of each parasitoid on the other $\left(\alpha_{p q}\right.$, and $\left.\alpha_{q p}\right)$ were estimated from the last experiment (this study). The carrying capacity has two different levels: $K_{\text {limited }}$ (with a mean of 43.5 plants and a mean number of 54.9 DBM adults found per cage) and $K_{\text {unlimited }}$ (with a mean of 215 plants and a mean of 101.2 DBM per cage). The following removal matrix, formerly was described as interaction strength $\left(I_{i, j}\right)$ between competitors by Paine (1992), was used to define the values of $\alpha_{p q}$ for our experimental data:

$\alpha_{p q}=\frac{N_{P Q}-N_{P Q-}}{N_{P Q}-N_{Q}}$

where $N_{P Q}$ is the abundance of $P$ (target) when the agent $(Q)$ is present, $N_{P Q-}$ is the abundance of the target when the agent is absent, and $N_{Q}$ is the abundance of the agent in absence of the target.

Values of parameters were then assigned to the ODEs (Eq. (1)) to derive the conditions for coexistence and the dynamic behaviors of the system. In case the two parasitoids cannot coexist due to competitive exclusion, we further analyzed the stability of the degraded system (i.e. one parasitoid system). The CA simulation was then used to analyze whether there is a minimum number of patches to ensure the coexistence of these two parasitoids and how the parasitoids distribute spatially in the lattices. Specifically, Wang and Keller (2002) have studied the movement of these two parasitoids, based on which we set the movement abilities as $e_{p}=0.01$ and $e_{q}=0.02$, and the searching areas as $n_{p}=8$ (Moore neighborhood) and $n_{q}=4$ (von Neumann neighborhood) (Hoekstra et al., 2010; Hui and Li, 2004) (Table 1). It is worth noting that seting the parameters to these values does not affect the overal results of spatial coexistence.

We ran this CA model on $70 \times 70$ lattices for 400 time steps to generate the spatial distribution of these three species (densities in patches). Each lattice in the CA model represents a patch of an average leaf size $\left(\sim 40 \mathrm{~cm}^{2}\right)$ and thus has $6.3 \mathrm{~cm} \times 6.3 \mathrm{~cm}$ in dimension. Considering the three-dimensional structure of canola (with 11 leaves per plant on average), a number of $5 \times 5$ lattices should 
Table 2

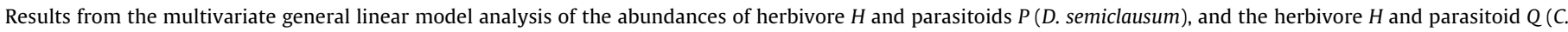
vestalis) under two levels of plant availability (i.e. limited and unlimited) and two coexistence situations (i.e. coexisting or not).

\begin{tabular}{|c|c|c|c|c|}
\hline Source & Dependent variables & Type III sum of squares & df & $F$ \\
\hline \multicolumn{5}{|l|}{ D. semiclausum } \\
\hline \multirow[t]{2}{*}{ Intercept } & $P$ & 12360.99 & 1 & $206.757^{* * *}$ \\
\hline & $H$ & 104186.93 & 1 & $1.712 \mathrm{E} 3^{* * *}$ \\
\hline \multirow[t]{2}{*}{ Coexistence } & $P$ & 27.46 & 1 & $0.459^{\mathrm{ns}}$ \\
\hline & $H$ & 109.41 & 1 & $1.798^{\text {ns }}$ \\
\hline \multirow[t]{2}{*}{ Plant } & $P$ & 924.16 & 1 & $15.458^{* *}$ \\
\hline & $H$ & 12800.66 & 1 & $210.396^{* * *}$ \\
\hline \multirow[t]{2}{*}{ Coexistence $\times$ Plant } & $P$ & 3.31 & 1 & $0.055^{\mathrm{ns}}$ \\
\hline & $H$ & 428.49 & 1 & $7.043^{*}$ \\
\hline \multicolumn{5}{|l|}{ C. vestalis } \\
\hline \multirow[t]{2}{*}{ Intercept } & $Q$ & 11259.33 & 1 & $92.843^{* * *}$ \\
\hline & $H$ & 84791.62 & 1 & $1.012 \mathrm{E} 3^{* * *}$ \\
\hline \multirow[t]{2}{*}{ Coexistence } & $Q$ & 146.17 & 1 & $1.205^{\mathrm{ns}}$ \\
\hline & $H$ & 446.48 & 1 & $5.330^{*}$ \\
\hline \multirow[t]{2}{*}{ Plant } & $Q$ & 642.62 & 1 & $5.299^{*}$ \\
\hline & $H$ & 7791.59 & 1 & $93.022^{* * *}$ \\
\hline \multirow[t]{2}{*}{ Coexistence $\times$ Plant } & $Q$ & 2.47 & 1 & $0.020^{\mathrm{ns}}$ \\
\hline & $H$ & 17.39 & 1 & $0.208^{\mathrm{ns}}$ \\
\hline $\begin{array}{l}{ }^{*} p<0.05 \\
p<0.01 \\
{ }_{* * *} \\
p<0.001 \\
\text { ns } \\
p>0.05\end{array}$ & & & & \\
\hline
\end{tabular}

represent all leaves of the two plants in a single pot and thus have a physical size of $31.5 \mathrm{~cm} \times 31.5 \mathrm{~cm}$ in the experiment, i.e. a width of $6.3 \mathrm{~cm}(=31.5 / 5)$ per patch. Consequently, the width of each lattice in the model is equal to $6.3 \mathrm{~cm}$ both in terms of leaf and pot dimensions, and the $70 \times 70$ lattices thus equal a habitat size of $14 \times 14$ pots (or $28 \times 28$ plants). We also ran the CA model with different number of lattices (from $1 \times 1$ to $15 \times 15$ ) to assess whether the coexistence of parasitoids can be affected by the habitat size (i.e. the number of patches). Each run started with randomly assigned initial values, and the proportion of the three scenarios (i.e. $P$ excluded, $Q$ excluded and the coexistence of $P$ and $Q$ ) were then estimated from 100 runs.

\section{Results}

The effects of plant availability on the equilibrium abundances of DBM and both parasitoids were significant but the coexistence of parasitoids $(P+Q)$ did not affect the abundance of parasitoids significantly (Table 2). There was also a significant effect of parasitoid coexistence on the herbivore abundance if parasitoid $Q$ (C. vestalis) remained from the competitive exclusion (Table 2 ).

According to the theory of limiting resources (MacArthur and Levins, 1964; Tilman, 1982), this tri-trophic system (Eq. (1)) does not have any positive stable equilibrium, meaning that the two parasitoids $(P$ and $Q)$ cannot coexist theoretically. The outcome of competitive exclusion relies on the following condition:

$\frac{P_{0}}{Q_{0}}>\frac{\gamma_{q} /\left(1+\gamma_{q} \mu_{q} H\right)}{\gamma_{p} /\left(1+\gamma_{p} \mu_{p} H\right)}$,

where $P_{0}$ and $Q_{0}$ are the initial population sizes of the two parasitoids. The right hand side of the above inequality stands for the ratio of the functional response of parasitoid $Q$ per herbivore $\left(\gamma_{q} /\left(1+\gamma_{q} \mu_{q} H\right)\right.$, i.e. the consumption rate of $H$ by $\left.Q\right)$ to the functional response of parasitoid $P$ per herbivore $\left(\gamma_{p} /\left(1+\gamma_{p} \mu_{p} H\right)\right.$, i.e. the consumption rate of $H$ by $P$ ). If this inequality holds, parasitoid $P$ will expel parasitoid $Q$; otherwise, parasitoid $Q$ will expel $P$. This suggests that the survivor from the parasitoid competition will entirely depend on the initial population consumption rate (=initial population size $\times$ per capita consumption rate). Apparently, competitive exclusion does not depend on the resource levels of plants $(R)$. Numerical simulations support this proposition that the above ratio (Eq. (4)) forms a divide in the phase plane (Fig. 2a and b).
Evidently, the ratio on the right hand of the above inequality is not really sensitive to the abundance of herbivores in our experiment (i.e. for the full range of $H$, the ratio remains around 0.58) (Fig. 2a and $b$ ).

Competitive exclusion of one parasitoid leads to a degraded system between the herbivore $(H)$ and the remaining parasitoid (Fig. 2c and d). For instance, for the degraded system that consists of herbivore $H$ and parasitoid $Q$, we have the following ODEs:

$$
\begin{aligned}
& \frac{1}{H} \frac{d H}{d T}=r\left(1-\frac{H}{K(R)}\right)-\frac{\gamma_{q} Q}{1+\gamma_{q} \mu_{q} H} \\
& \frac{1}{Q} \frac{d Q}{d T}=\frac{\gamma_{q} H}{1+\gamma_{q} \mu_{q} H}-m_{q}
\end{aligned}
$$

This degraded system only has a single non-negative equilibrium $\left(H^{*}, Q^{*}\right)$ :

$$
\left(\frac{m_{q}}{\gamma_{q}\left(1-\mu_{q} m_{q}\right)}, \frac{r\left(1-H^{*} / K\right)}{\gamma_{q}\left(1-\mu_{q} m_{q}\right)}\right)
$$

The herbivore equilibrium does not depend on its own vital parameters ( $r$ and $K$ ) that could potentially be affected by the plant resource levels but, rather, depends on the vital parameters of the parasitoid. This suggests that herbivore density is largely controlled by the parasitoid rather than by the level of plant availability. In contrast, the parasitoid equilibrium depends on both its own vital parameters and those of the herbivore that could be potentially affected by the level of plant availability. This indicates the existence of a bottom-up regulation of parasitoid density by the host plant.

Although the two parasitoids cannot coexist on a single herbivore population, the CA simulation demonstrated that they could spatially coexist, forming local clusters of degraded systems (Fig. 3). Both parasitoids exhibited spiral structures when the carrying capacity ( $K=50$ and 100$)$ and the population growth rate of DBM were high ( $r=0.304$ and 0.6 ) (Fig. 3). Measured by Moran's I index with a negative exponential distance weight (see Appendix S1 in Hui et al., 2011), the level of patchiness in Fig. 3b increased significantly from 0.215 in plot 4 to 0.257 in plot $6,0.271$ in plot 8 and 0.337 in plot $10(t>82.4, P<0.001)$; Moran's $I$ index in Fig. 3c also significantly increased from 0.276 in plot 4 to 0.307 in plot $6,0.319$ in plot 8 and 0.32 in plot $10(t>60.4, P<0.001)$. The densities of parasitoid $P$ and $Q$ in these patches showed a 


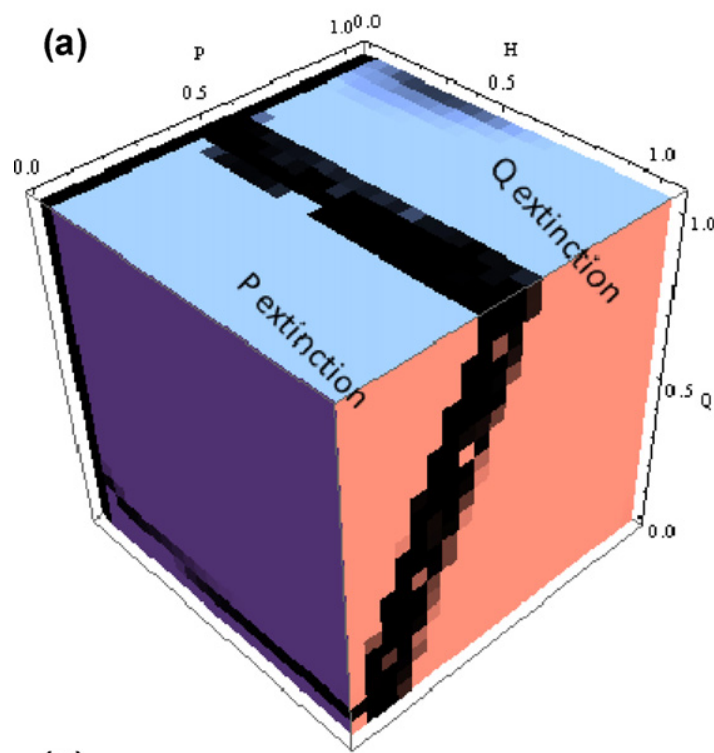

(c)

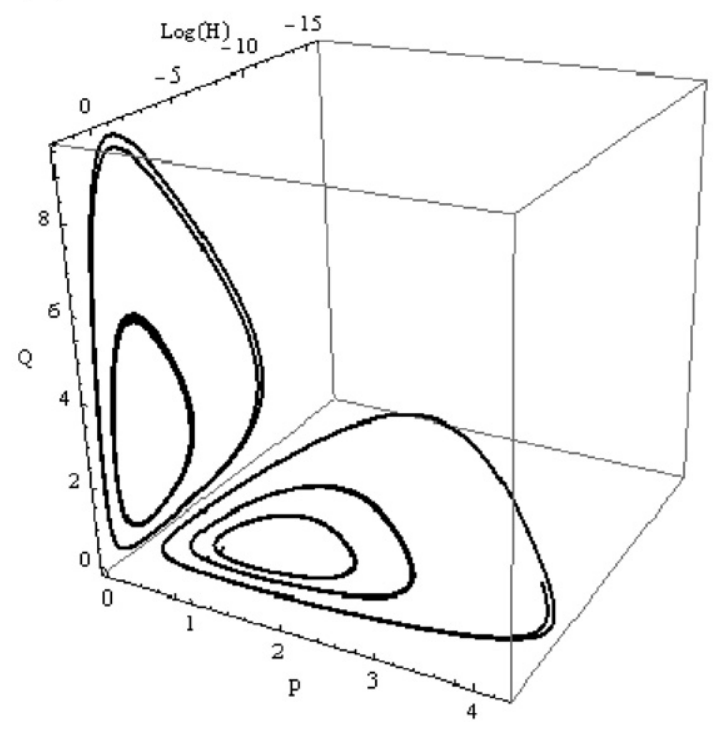

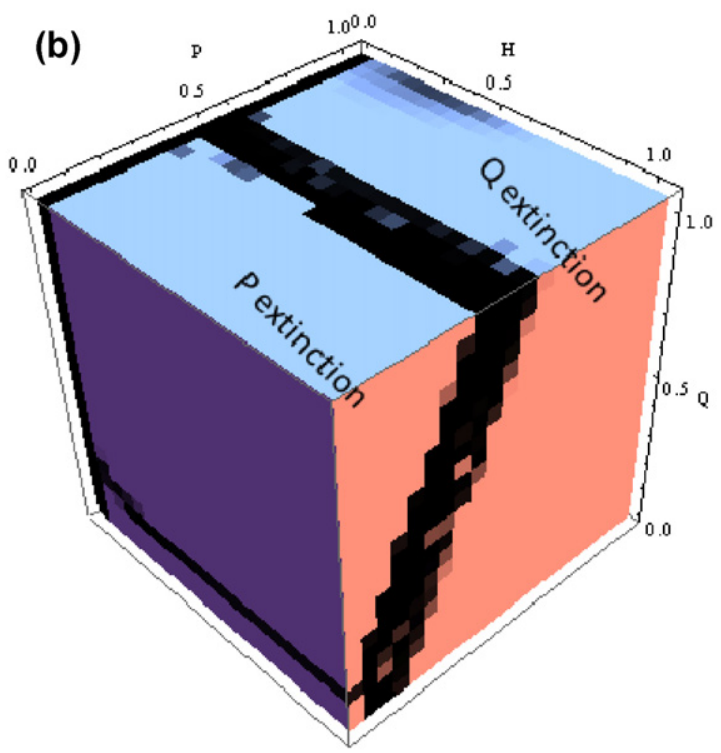

(d)

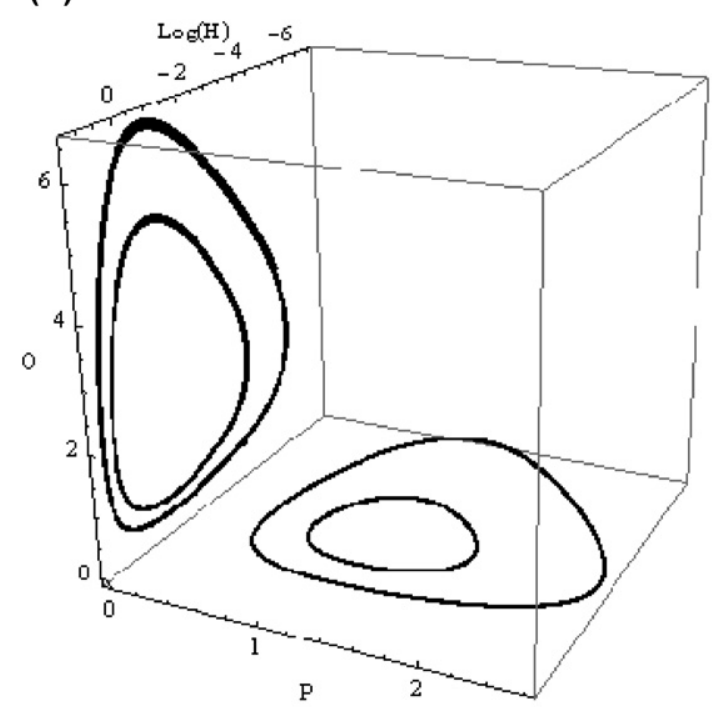

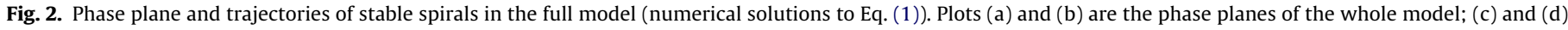
are illustrations of trajectories from different initial conditions; (a) and (c) are results for plant limited treatment; (b) and (d) are results for plant unlimited treatment.

negative correlation (e.g. compare b9 with c9 in Fig. $3, \ln (P)$ vs. $\ln (Q)$ for $P$ and $Q>0.0001: r=-0.837, P<0.001$; similarly, compare b11 with c11: $r=-0.803, P<0.001)$. Two cases were further resized to demonstrate the details of the negative correlation in the distributions of $P$ and $Q$ (Fig. 3).

With an increase of the patch number, the proportions of the persistence of parasitoid $P$ (and also $Q$ ) and the coexistence of $P$ and $Q$ reached $100 \%$ when the habitat consists of more than $9 \times 9$ patches (Fig. 4 a). The periodic oscillation in degraded systems (Fig. 2c and d) also became damped oscillations (Fig. 4b).

\section{Discussion}

Evidently, the herbivore and both parasitoids had higher abundances under the unlimited plant treatment (i.e. high level of plant availability). Abundances of parasitoid $P$ and $Q$ when coexisting did not differ from the abundances when they were alone in the system, suggesting that the coexistence of these two parasitoids does not bring each other benefit or cost. Therefore, in contrast to
Amarasekare's (2002) assertion, there is no effect of interference on the competitive coexistence. The fact that parasitoid $P$ and $Q$ are able to coexist in a cage for at least 8-9 generations suggests mechanisms other than interference could be at work.

Heterogeneous habitat and temporal niche separation are potential mechanisms fostering coexistence of two specialist parasitoids on a single host (Hackett-Jones et al., 2009; Hassell et al., 1991). For instance, specialist parasitoids can coexist if they prefer different oviposition sites or if they have an additional relationship of intraguild predation (Borer, 2002; Borer et al., 2004). Our results showed that the two parasitoids with different searching efficiencies on the same host cannot coexist at a single location unless considering different movement abilities and search area sizes (e.g. Shi et al., 2004; Talekar and Yang, 1991; Wang and Keller, 2002). These two larval parasitoids can coexist in the CA simulation and form spatially non-overlapping distributions, especially when the vital parameters of DBM $(r$ and $K$ ) are high. These nonoverlapping distributions have been widely reported as from strong competition of ecologically similar species (e.g. MacArthur, 1958; 


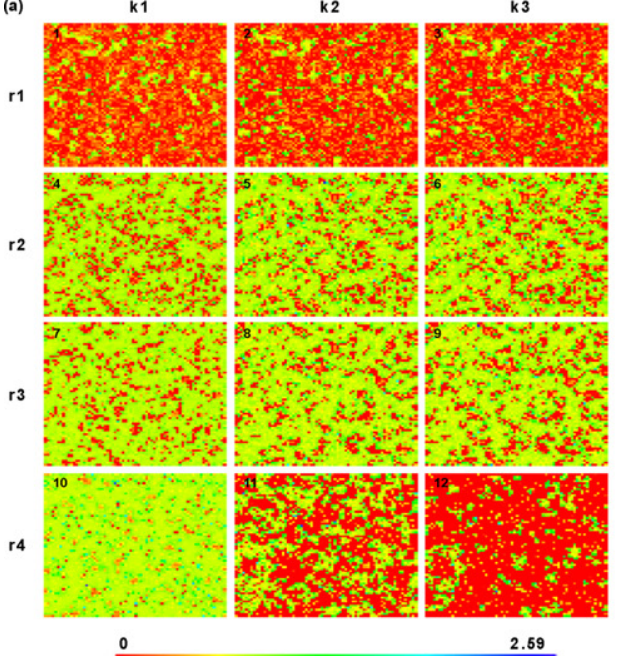

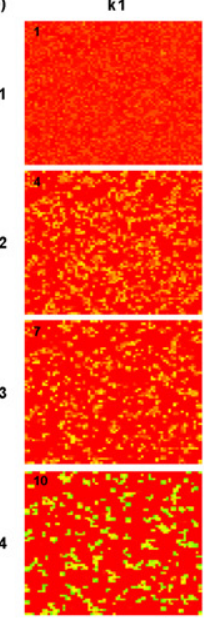
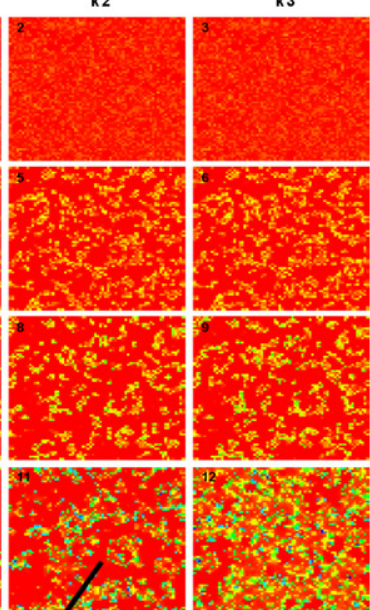

(c)

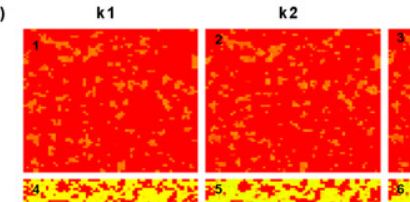

r2
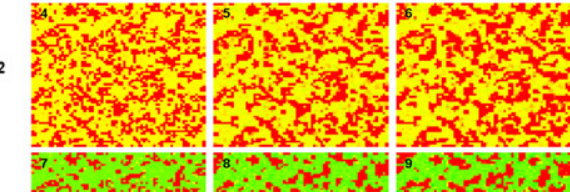

r3

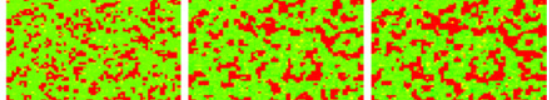

\section{it:}

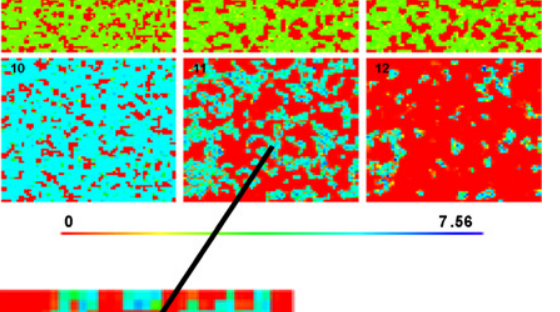

11.38
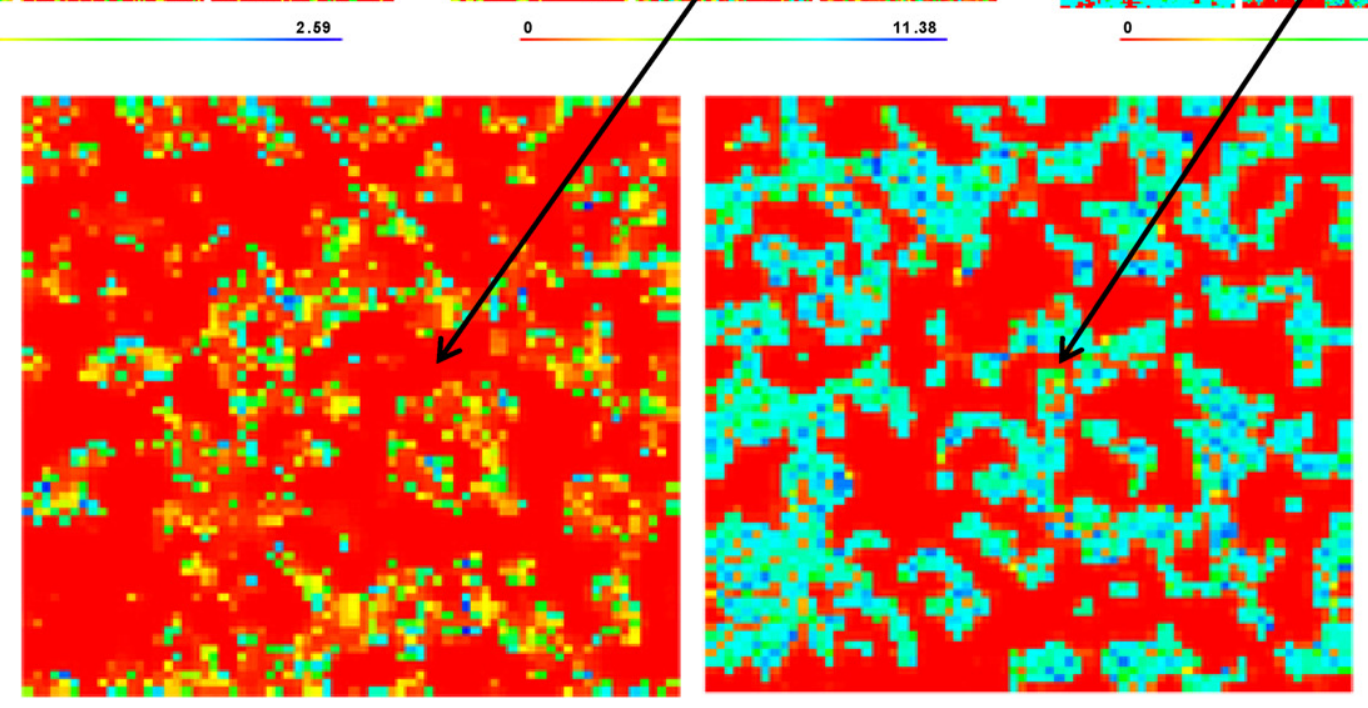

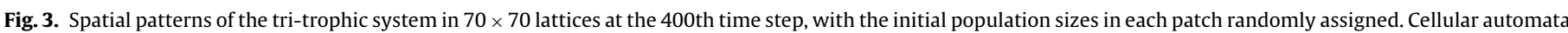

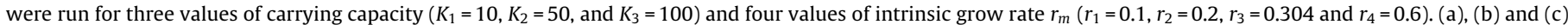

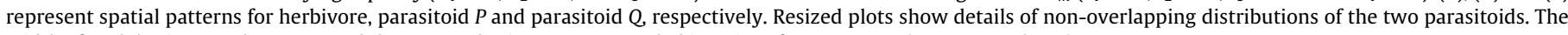
width of each lattice equals $6.3 \mathrm{~cm}$, and the $70 \times 70$ lattices represent a habitat size of $14 \times 14$ pots (or $28 \times 28$ plants).

Schoener, 1974; Wilson, 1984). Therefore, there could be a local density-dependent regulation by both parasitoids, consistent with the pattern of percent parasitism in Hassell et al. (1991).

It is worth noting that the outcome of the competitive exclusion depends on the functional response ratio of the two parasitoids (Eq. (4)). This ratio consists of attack rates and handling times of two parasitoids, suggesting that bottom-up forces (plant availability) which contribute to the fitness of the herbivore $(r$ and $K$ ) do not affect the outcome of parasitoid competition especially in a local habitat patch. However, the statistical analysis indicated a significant effect of plant availability on the abundance of both herbivore and parasitoids (Table 2), suggesting that the bottomup force (plant availability) only affects the material flow but not the topological structure of this tri-trophic food web.

The model suggests that the coexistence of multiple parasitoids on one host is locally impossible but spatially feasible. In comparison to other models of the same sort, we further identified a few factors that could potentially intensify the competitive exclusion. Firstly, Hackett-Jones et al. (2009) have demonstrated a destabilizing effect of the type II functional response in a Nicholson-Bailey model which can lead to the competitive exclusion of one parasitoid species. Briggs (1993) has analyzed a similar system using stage-structured delay differential equations with a type I functional response and found similar results that either parasitoid $P$ or $Q$ may expel the other depending on initial conditions. However, using a similar model with the type I functional response, Amarasekare (2002) has demonstrated the coexistence of two consumers on a single resource and attributes the coexistence to the potential beneficial interference between the two consumers. This suggests that the forms of functional responses might not be the real reason for competitive exclusion. Clearly, more investigation is needed to clarify the role of functional responses in the stability and structure of food webs.

Secondly, both non-stage and stage-structured Lotka-Volterra models of homogenous populations have exhibited competitive exclusion of species by the most efficient competitors (Briggs, 1993; Mills and Getz, 1996). Our results support that spatial heterogeneity induced by both the patch habitat and the patchily distributed DBMs in the CA simulation as a promotor of competitive coexistence. It is further evident that there exists a minium habitat size to ensure species persistence and coexistence (Fig. 4a), consistent with the observation from Zhao et al. (2012). Evidently, damped oscillations in Fig. 4b indicated that large habitat size with multiple patches can further stabilize the dynamics of the system and promote the coexistence of these two competing parasitoids. The effects of environmental and spatial heterogeneity formed by both host plants and herbivores on the coexistence of parasitoids at the third-trophic level warrants further investigation. 

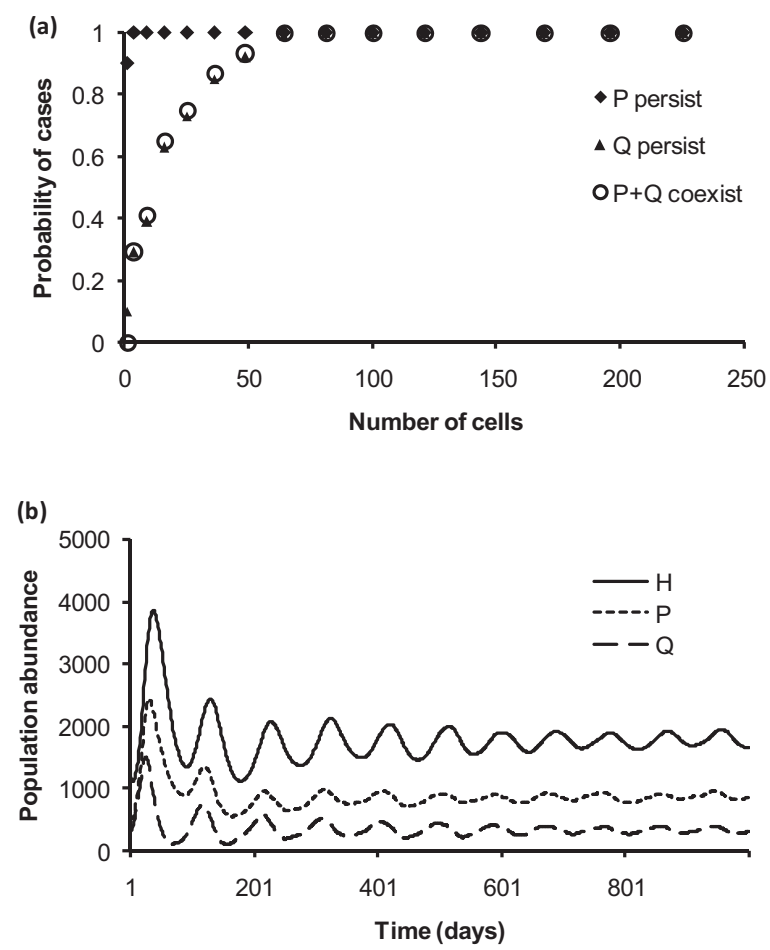

Fig. 4. (a) The probability of different cases at the 400th time step as a function of the number of cells (patches) in the cellular automaton, with initial population sizes randomly assigned. Coexistence can be guaranteed when the number of cells is about $9 \times 9$. (b) Damped oscillations of population sizes on a habitat of $15 \times 15$ lattices.

Overall, our results support that $D$. semiclausum is a more effective parasitoid than $C$. vestalis (Wang and Keller, 2002; but see Shi et al., 2004). Moreover, the competitive coexistence and exclusion of parasitoids is sensitive to the initial population sizes, functional response per herbivore and the patchiness of plant and herbivores, but not the level of plant availability. Other bottom up factors, such as the availability of refuge and the complexity of plant morphology that could potentially affect the persistence and coexistence of DBM parasitoids need to be examined in future studies.

\section{Acknowledgements}

We are grateful to the DST-NRF Centre of Excellence for Invasion Biology (CIB) at Stellenbosch University for logistic help. We wish to thank Myron P. Zalucki for his useful comments on early draft of the manuscript. This work was funded by Tarbiat Modares University and partly by Intergrated Pest and Disease Management of Oil Crops of Iran.

\section{Appendix A. Supplementary data}

Supplementary data associated with this article can be found, in the online version, at http://dx.doi.org/10.1016/ j.ecolmodel.2012.06.033.

\section{References}

Adler, P.B., HilleRisLambers, J., Levine, J.M., 2007. A niche for neutrality. Ecology Letters 10, 95-104.

Amarasekare, P., 2002. Interference competition and species coexistence. Proceedings of the Royal Society B 269, 2541-2550.

Amarasekare, P., 2007. Trade-offs, temporal variation, and species coexistence in communities with intraguild predation. Ecology 88, 2720-2728.

Bach, C.E., 1988. Effects of host plant patch size on herbivore density: patterns. Ecology 69, 1090-1102.
Bonsall, M.B., Hassell, M.P., Asefa, G., 2002. Ecological trade-offs, resource partitioning, and coexistence in a host-parasitoid assemblage. Ecology 83, 925-934.

Borer, E.T., 2002. Intraguild predation in larval parasitoids: implications for coexistence. Journal of Animal Ecology 71, 957-965.

Borer, E.T., Murdoch, W.W., Swarbrick, S.L., 2004. Parasitoid coexistence: linking spatial field patterns with mechanism. Ecology 85, 667-678.

Bottrell, D.G., Barbosa, P., Gould, F., 1998. Manipulating natural enemies by plant variety selection and modification: a realistic strategy? Annual Review of Entomology 43, 347-367.

Briggs, C.J., 1993. Competition among parasitoid species on a stage-structured host and its effect on host suppression. American Naturalist 141, 372-397.

Chesson, P.L., Case, T.J., 1986. Overview: nonequilibrium community theories chance variability history and coexistence. In: Diamond, J., Case, T.J. (Eds.), Community Ecology. Harper and Row, Philadelphia.

Comins, H.N., Hassell, M.P., 1996. Persistence of multispecies host-parasitoid interactions in spatially distributed models with local dispersal. Journal of Theoretical Biology 183, 19-28.

Hackett-Jones, E., Cobbold, C., White, A., 2009. Coexistence of multiple parasitoids on a single host due to differences in parasitoid phenology. Theoretical Ecology 2, 19-31.

Hassell, M.P., May, R.M., Pacala, S.W., Chesson, P.L., 1991. The persistence of host-parasitoid associations in patchy environments. I. A general criterion. American Naturalist 138, 568-583.

Hastings, A., 1980. Disturbance, coexistence, history, and competition for space. Theoretical Population Biology 18, 363-373.

Hemerik, L., 2007. Feeding status of the parasitoid Diadegma semiclausum affects biological control of Plutella xylostella: a simulation study. In: Proc. Neth. Entomol. Soc. Meet., Netherlands.

Hoekstra, A.G., Kroc, J., Sloot, P.M.A., 2010. Simulating Complex Systems by Cellular Automata. Springer-Verlag, Berlin.

Holling, C.S., 1959. Some characteristics of simple types of predation and parasitism. Canadian Entomologist 91, 385-398.

Hui, C., Foxcroft, L.C., Richardson, D.M., MacFadyen, S., 2011. Defining optimal sampling effort for large-scale monitoring of invasive alien plants: a Bayesian method for estimating abundance and distribution. Journal of Applied Ecology $48,768-776$.

Hui, C., Li, Z., 2003. Dynamical complexity and metapopulation persistence. Ecological Modelling 164, 201-209.

Hui, C., Li, Z., 2004. Distribution patterns of metapopulation determined by Allee effects. Population Ecology 46, 55-63.

MacArthur, R.H., 1958. Population ecology of some warblers of northeastern coniferous forests. Ecology 39, 599-619.

MacArthur, R.H., Levins, R., 1964. Competition, habitat selection, and character displacement in a patchy environment. Proceedings of the National Academy of Sciences of the United States of America 51, 1207-2121.

McGlade, J.M., 1999. Advanced Ecological Theory: Principles and Applications. Blackwell Scientific Publications, Oxford.

Mills, N.J., Getz, W.M., 1996. Modelling the biological control of insect pests: a review of host-parasitoid models. Ecological Modelling 92, 121-143.

Mitsunaga, T., Shimoda, T., Yano, E., 2004. Influence of food supply on longevity and parasitization ability of a larval endoparasitoid, Cotesia plutellae (Hymenoptera, Braconidae). Applied Entomology and Zoology 39, 691-697.

Nofemela, R.S., 2004. Studies on Parasitoids of the Diamondback Moth, Plutella xylostella (L.) (Lepidoptera: Plutellidae) in South Africa. Rhodes University, Grahamstown, South Africa.

Ohgushi, T., 2007. Nontrophic, indirect interaction webs of herbivorous insects. In: Ohgushi, T., Craig, T.P., Price, P.W. (Eds.), Ecological Communities: Plant Mediation in Indirect Interaction Webs. Cambridge University Press, Cambridge, UK, pp. 221-245.

Paine, R.T., 1992. Food-web analysis through field measurement of per capita interaction strength. Nature 355, 73-75.

Ramanantoanina, A., Hui, C., Ouhinou, A., 2011. Effects of density-dependent dispersal behaviours on the spread and spatial patterns of range expansion in predator-prey metapopulations. Ecological Modelling 222, 3524-3530.

Schoener, T.W., 1974. Resource partitioning in ecological communities. Science 185, 27-39.

Schoener, T.W., 1982. The controversy over interspecific competition. American Scientist 70, 586-595.

Shi, Z.H., Li, Q.B., Li, X., 2004. Interspecific competition between Diadegma semiclausum Hellén (Hym., Ichneumonidae) and Cotesia plutellae (Kurdjumov)(Hym., Braconidae) in parasitizing Plutella xylostella (L) (Lep., Plutellidea). Journal of Applied Entomology 128, 437-444.

Soufbaf, M., Fathipour, Y., Karimzadeh, J., Zalucki, M.P., 2010. Bottom-up effect of different host plants on Plutella xylostella (Lepidoptera: Plutellidae): a life-table study on canola. Journal of Economic Entomology 103, 2019-2027.

Soufbaf, M., Fathipour, Y., Zalucki, M.P., Hui, C., 2012. Importance of primary metabolites in canola in mediating interactions between a specialist leaf-feeding insect and its specialist solitary endoparasitoid. Arthropod-Plant Interactions 6 , 241-250.

SPSS, 2008. SPSS Base 16.0.2 for Windows User's Guide. SPSS Inc., Chicago, IL.

Talekar, N.S., Yang, J.C., 1991. Characteristic of parasitism of diamondback moth by two larval parasites. Entomophaga 36, 95-104.

Talekar, N.S., Yang, J.C., 1993. Influence of crucifer cropping system on the parasitism of Plutella xylostella (Lep., Yponomeutidae) by Cotesia plutellae (Hym., Braconidae) and Diadegma semiclausum (Hym., Ichneumonidae). Entomophaga $38,541-550$. 
Tilman, D., 1982. Resource Competition and Community Structure of Plant Communities. Princeton University Press, Princeton.

Walls, S.C., 1990. Interference competition in postmetamorphic salamanders: interspecific differences in aggression by coexisting species. Ecology 71 , 307-314.

Wang, X.G., Keller, M.A., 2002. A comparison of the host-searching efficiency of two larval parasitoids of Plutella xylostella. Ecological Entomology 27, 105-114.
Werner, E.E., Peacor, S.D., 2003. A review of trait-mediated indirect interactions in ecological communities. Ecology 84, 1083-1100.

Wilson, W.H., 1984. Non-overlapping distributions of spionid polychaetes: the relative importance of habitat and competition. Journal of Experimental Marine Biology and Ecology 75, 119-127.

Zhao, Z.-H., He, D.-H., Hui, C., 2012. From the inverse density-area relationship to the minimum patch size of a host-parasitoid system. Ecological Research 27 , 303-309. 